\title{
GESTIÓN DE RIESGOS DE MERCADO Y SU IMPACTO EN LA ESTABILIDAD DEL SISTEMA BANCARIO PERUANO
}

\author{
MARKET RISK MANAGEMENT AND ITS IMPACT ON THE STABILITY OF THE \\ PERUVIAN BANKING SYSTEM
}

\author{
Manuel Alberto Hidalgo Tupia \\ Universidad Nacional Mayor de San Marcos \\ Lima, Perú \\ ORCID: https://orcid.org/0000-0002-9644-2406 \\ Correo electrónico: mhidalgot@unmsm.edu.pe
}

\section{RESUMEN}

Objetivo: Determinar el nivel de impacto de la Gestión del Riesgo de Mercado conforme a las normas de la Superintendencia de Banca, Seguros (SBS) y AFP en la estabilidad de las empresas bancarias peruanas. Método: La investigación fue no experimental, usó un diseño trasversal, utilizando como instrumentos de recolección de información el análisis documental y la encuesta. Resultados: Mostraron una relación significativa entre la aplicación del Sistema de Gestión de Riesgo de Mercado (SGRM) y la estabilidad del sistema bancario del Perú, evidenciando, asimismo, diferencias en el impacto de cada componente del SGRM. Conclusiones: Se determinó que la aplicación del SGRM influye positivamente en la estabilidad del sistema bancario peruano, indicándose factores que pueden afectar dicha influencia.

Palabras clave: Banca; riesgo bancario; riesgo de mercado; supervisión bancaria.

\begin{abstract}
Objective: Determine the level of impact of Market Risk Management in accordance with the risk management standards of the Superintendency of Banking, Insurance (SBS) and AFP on the stability of Peruvian banking companies. Method: The research was non-experimental, it used a transverse design, using documentary analysis and survey as information gathering tools. Results: They showed a significant relationship between the application of the Market Risk Management System (SGRM) and the stability of the banking system in Peru, showed, differences in the impact of each component of the SGRM. Conclusions: It was determined that the implementation of the SGRM positively influences the stability of the Peruvian Banking System, indicating factors that may affect that influence.
\end{abstract}

Keywords: Banking; banking risk; market risk; banking supervision.

(c) Los autores. Este artículo es publicado por la Revista Quipukamayoc, Universidad Nacional Mayor de San Marcos. Este es un artículo de acceso abierto, distribuido bajo los términos de la Licencia Creative Commons Atribución-NoComercial-Compartirlgual 4.0 Internacional.(http://creativecommons.org/licenses/by-nc-sa/4.0/), que permite el uso no comercial, distribución y reproducción en cualquier medio, siempre que la obra original sea debidamente citadas. 


\section{INTRODUCCIÓN}

No cabe duda de la gravitación de la estabilidad del sistema bancario en la estabilidad económica y el crecimiento de un país, en el caso de su ausencia, como se ha podido apreciar en la larga secuela de crisis financieras ocurridas en el mundo. (Mishkin, 2014) Crisis financieras que poseen un efecto sistémico trasfronterizo mucho mayor ahora que en las crisis precedentes, como la crisis de 1929 en los Estados Unidos. Se ha enfatizado en la relación y consecución histórica de las crisis: bancaria, financiera, fiscal, monetaria y económica. (Reinhart y Rogoff, 2011)

Esta ha sido la razón fundamental por la cual los principales bancos, los gobiernos y los organismos multilaterales han hecho grandes esfuerzos, en especial, desde 1988, por prevenir este tipo de crisis originadas en los sistemas bancarios, a través de la regulación y la supervisión global. Específicamente, el esfuerzo se concentró en el Comité Internacional de Supervisión Bancaria de Basilea (CISBB), promovida por el Banco de Liquidaciones Internacionales, establecido en dicha ciudad Suiza, que, con el aporte inicial de los bancos centrales de las principales naciones industriales estableció en 2006 la primera versión de los Principios de la Supervisión Bancaria Eficaz (Comité de Supervisión Bancaria de Basilea, 2018) y posteriormente los Acuerdos de Capital de Basilea (Comité de Supervisión Bancaria de Basilea, 2001), que ya tiene tres versiones, con la adhesión de casi todas las economías del mundo, para establecer un marco global común de regulación y supervisión bancaria.

El Perú ha adoptado el marco regulatorio y de supervisión establecido en los Acuerdos de Capital de Basilea, incluyéndolo en su legislación (Ley 26702, 1996), aplicada a las empresas bancarias peruanas, y supervisadas por la SBS. El sistema bancario peruano está compuesto por 16 bancos, sujetos a la supervisión de la SBS en el año 2017, para el análisis respectivo, con características muy poco homogéneas, es decir, con una concentración muy alta en términos de depósitos, créditos y activos, en los 4 primeros bancos del sistema (BCP, BBVA Continental, Scotiabank e Interbank).

El Sistema Bancario peruano presenta una estabilidad notable desde 2001, cuando terminó la última crisis bancaria en el país, con niveles de morosidad moderados, pero significativamente mayores que el promedio de la región. Más aún, las sucesivas advertencias del Banco Central de Reserva del Perú, BCRP, (Alva, 2016), así como de la propia Asociación de Bancos muestran una concentración de la morosidad en ciertos sectores desde el punto de vista del crédito, como por ejemplo los créditos de consumo para las familias, y los créditos a las micro y pequeñas empresas. Esta situación de morosidad latente, unida a las características de concentración de la banca peruana, hace que la estabilidad del sistema bancario presente riesgos a prevenir.

De los tres riesgos generales que enfocan los Acuerdos de Basilea -de crédito, de mercado y operacionales -(Comité de Supervisión Bancaria de Basilea, 2001) aunque el riesgo de crédito tiene el efecto más inmediato en la estabilidad de las empresas bancarias, el riesgo de mercado, pese a su relativa menor inmediatez para la estabilidad bancaria, se acrecienta en forma dramática en el contexto actual de globalización junto a la mayor volatilidad de los distintos mercados no financieros y financieros. Por ello el FMI (Fondo Monetario Internacional, 2013) se ha visto obligado a establecer un seguimiento especial a la estabilidad financiera, a través del periódico Reporte de la Estabilidad Financiera. Asimismo el Banco Central de Reserva del Perú hace un seguimiento similar al del FMI para Perú; además del tratamiento especial de la SBS (SBS, 2017).

Esta ha sido la motivación central para la elección del tema de investigación, la pertinencia de las disposiciones sobre Gestión de Riesgo de Mercado en el sistema bancario peruano para su estabilidad. Se enfoca en la Gestión de Riesgo de Mercado, además, por ser un tema poco estudiado, con sus 5 componentes: gestión aplicada a divisas, a acciones, a bonos, a créditos interbancarios y a derivados financieros.

Estudios previos referente al riesgo en general aplicado a la banca peruana, tienen como aporte más cercano al de Saldaña, quien en un estudio del periodo 2001 - 2006, concluye que en un mercado oligopolizado que ya presenta vulnerabilidad, cualquier problema en las empresas, sea coyuntural o estructural, se trasmite inmediatamente al mercado. (Saldaña, 2009)

Lizarzaburu, Berggrun y Quispe (2012) analiza la gestión de los riesgos financieros en general en un banco peruano, abordan la gestión de riesgo de crédito y operacional, mas no de mercado. Sin embargo, el estudio aporta una serie de conceptos teóricos pertinentes, bajo la misma normatividad proveniente de los Acuerdos de Capital de Basilea.

Otra investigación se relacionó con un aspecto relativo al riesgo de crédito, la morosidad, en un segmento de los clientes bancarios, como lo es el sector MYPE en Perú. Analiza el desenvolvimiento de la morosidad en el período 2010 y 2014, en dicho segmento, que llama la atención por su nivel elevado de morosidad respecto a los demás segmentos, como el corporativo, gran y mediana empresa. Se enfoca en especial en las políticas de calificación bancaria y gestión de riesgo de crédito. (Huertas, 2015) 
Castro (2011) se refiere a los riesgos del sistema bancario peruano, enfocando en especial la crisis de 2001, aludiendo a los riesgos de crédito y cambiario, que se considera uno de los componentes del riesgo de mercado. Concluye que la concentración de los créditos a empresas vinculadas a los accionistas principales de los bancos quebrados (relacionados al riesgo crediticio) y la dolarización de los créditos coadyuvaron a la crisis de ese año.

Una investigación relativa a la Banca de México, López (2018) incluye un enfoque sobre el riesgo de crédito y también del riesgo de mercado, enfatizando que la proliferación de nuevos instrumentos financieros como los derivados (futuros, swaps y opciones) y la volatilidad de los factores que afectan su valoración, requieren nuevas medidas para disminuir el riesgo sistémico. Propone medidas internas, a aplicarse por el Banco de México y la Secretaria de Hacienda y Crédito Público, y externas, conforme a lo propuesto por el Comité de Basilea.

En España, Gutierrez (2013), analiza la debilidad de la regulación y supervisión bancaria (incluyendo el sistema de gestión de riesgos de mercado) ante el carácter sistémico de los riesgos más gravitantes resaltados durante los últimos quinquenios, aspectos en que coincide con el enfoque de Freixas (Freixas, 1990.).

La investigación de Morales se enfoca al conjunto del sistema bancario peruano, identifica, que determinados sectores de la economía, como las microempresas (MYPE) no han sido incluidos en el sistema bancario, y señala que las tasas de interés elevadas son una de las causas. (Morales, 2014)

Sobre el sistema bancario internacional, el análisis ha sido bastante amplio, (Mishkin, 2014), en especial, de los efectos de la globalización en las finanzas y el consecuente incremento del riesgo sistémico (Gutierrez, 2013), además de las consecuencias macroeconómicas, nacionales y globales, de las crisis iniciadas en el sector bancario (Wolf, 2015), (Flores y Hidalgo, 2011) como la de Estados Unidos, que inicia en los mercados financieros y repercute en el sector bancario (Congreso de Los Estados Unidos, 2011). La relación entre las crisis financiera, monetaria, bancaria, fiscal y externa, la analizan asimismo varios autores (Reinhart y Rogoff, 2011; Marín y Valle, 2009).

Sobre la regulación bancaria internacional, de los Acuerdos de Capital de Basilea, los autores Apostolik, Donohue, y Went (2011); Holand (2016); Freixas (1990); Partal y Gómez (2011); Marín y Valle (2009) han aportado un análisis exhaustivo, bajo el marco de la creciente volatilidad de los mercados financieros.

Sobre la estabilidad de las empresas bancarias en el Perú (Alva y Rivas, 2012) y sus características de concentración, se ha incorporado el análisis de los grupos de poder económico (Durand, 2017) como una categoría explicativa. Además, el seguimiento de sus principales indicadores lo aporta la propia Superintendencia de Banca, Seguros y AFP (SBS, 2017).

\section{MATERIALES Y MÉTODOS}

La investigación es de tipo no experimental, de diseño transversal. La unidad de análisis lo constituyen las Instituciones del sistema bancario peruano. La población está constituida por las 16 instituciones bancarias locales. Se consideró asimismo una muestra de expertos, elegida con los siguientes criterios: 1) Expertos en Administración bancaria, 2) Investigadores con experiencia en los temas de finanzas. Las técnicas de recolección de datos fueron la encuesta, y complementariamente el análisis documental.

La elección de la técnica principal (encuesta) se debió a las limitaciones en la información disponible de la SBS, debido a que las normas regulatorias permiten a cada banco establecer estándares propios, basados en sus propias políticas, por lo cual, los indicadores de cada banco, al tener estándares diferentes, no son comparables.

Pese a los esfuerzos realizados, no fue posible obtener valoraciones propias de los bancos sobre como el SGRM impacta en su estabilidad, probablemente, por un concepto de secreto empresarial. Esta limitación es similar a la mencionada por otros autores en relación a los bancos y su información sobre los créditos vencidos. (Reinhart y Rogoff, 2011)

\section{RESULTADOS}

Formulando una prueba empírica sobre el carácter oligopólico del sistema bancario del Perú, se optó por aplicar el coeficiente de Gini, precisamente para medir la igualdad o desigualdad de ingresos o patrimonio de agentes económicos. Calculando el coeficiente de Gini respecto al patrimonio, este alcanza un 0,66 lo cual indica un alto nivel de concentración. El coeficiente de Gini varía entre el 0 (distribución equitativa) hasta 1 (distribución totalmente inequitativa), por lo cual dicho valor indica una concentración del patrimonio bancario muy marcado.

Asimismo, se consideró la morosidad del sistema bancario local. La morosidad de los créditos bancarios en el Perú fue creciente, como se aprecia en la figura 1.

En la tabla 1 se presenta una síntesis de los resultados encontrados, al medir, en la opinión de los expertos seleccionados, la incidencia del Riesgo del Mercado en el sistema bancario peruano, mostrándose los promedios en cada una de las 5 dimensiones. 


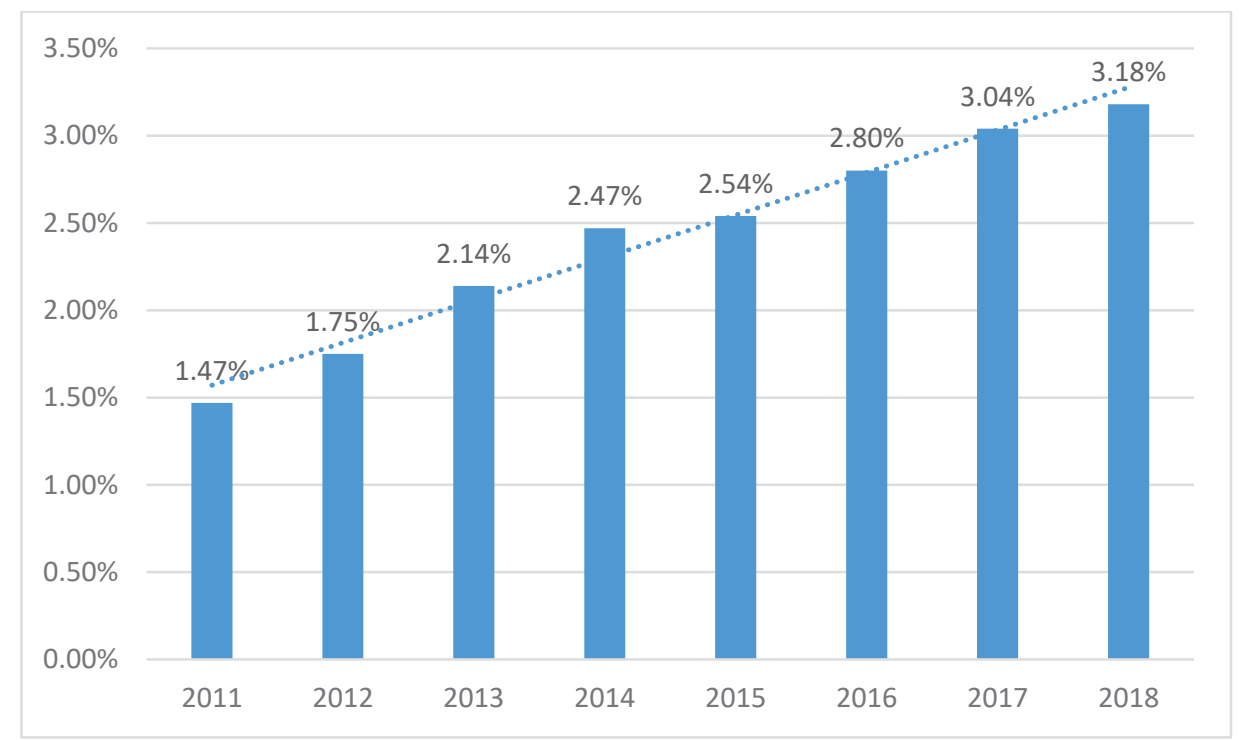

Figura 1. Evolución de la morosidad de los créditos bancarios en el Perú.

Fuente: Elaboración propia.

Tabla 1

Incidencia del Riesgo de Mercado en las empresas bancarias- Perú

\begin{tabular}{lc}
\hline Tipo & Índice \\
\hline Riesgo de Mercado relacionado a divisas & 0,541 \\
Riesgo de Mercado relacionado a acciones & 0,205 \\
Riesgo de Mercado relacionado a bonos & 0,453 \\
Riesgo de Mercado relacionado a créditos & 0,50025 \\
Riesgo de Mercado relacionado a derivados & 0,4825 \\
Riesgo de mercado relacionado a factores sistémicos & 0,599 \\
\hline
\end{tabular}

Fuente. Aplicación de encuesta. Elaboración propia

En el cual la incidencia total equivale a 1, y la incidencia nula a 0.

Los resultados se presentan en la figura 2 que se muestra a continuación.

La Gestión de Riesgos de Mercado (GRM) respecto a divisas presenta un impacto más alto, mientras la GRM relativo a acciones, el más bajo.

Como se puede apreciar, los resultados presentan las siguientes características:

I. Muestran una correlación positiva,

II. Muestran una correlación regular, no alta;

III. Muestran una correlación diferenciada, entre 0,42 la mínima, hasta 0,599 la máxima.
Asimismo, la relación es significativa, conforme a la prueba de hipótesis planteada, midiendo la correlación con el índice R cuadrado de Pearson. Por lo cual, la correlación es positiva, pero no es fuerte, como lo debería ser conforme a las bases teóricas. La pregunta que corresponde es la razón por la cual la correlación no es fuerte, como se discutirá más adelante.

Para el análisis descriptivo de frecuencias se presenta a continuación los resultados individuales de los 5 componentes del SGRM, como sigue:

En la figura 3 se observa que el 46,51\% de las respuestas se concentra en la categoría Regular, en tanto que existe una tendencia compartida de $27,91 \%$ a ser Mucho, con una tendencia a ser Poco en un 20,93\% de índice; por último, un índice de $4,65 \%$ a la categoría Totalmente. Siendo el 


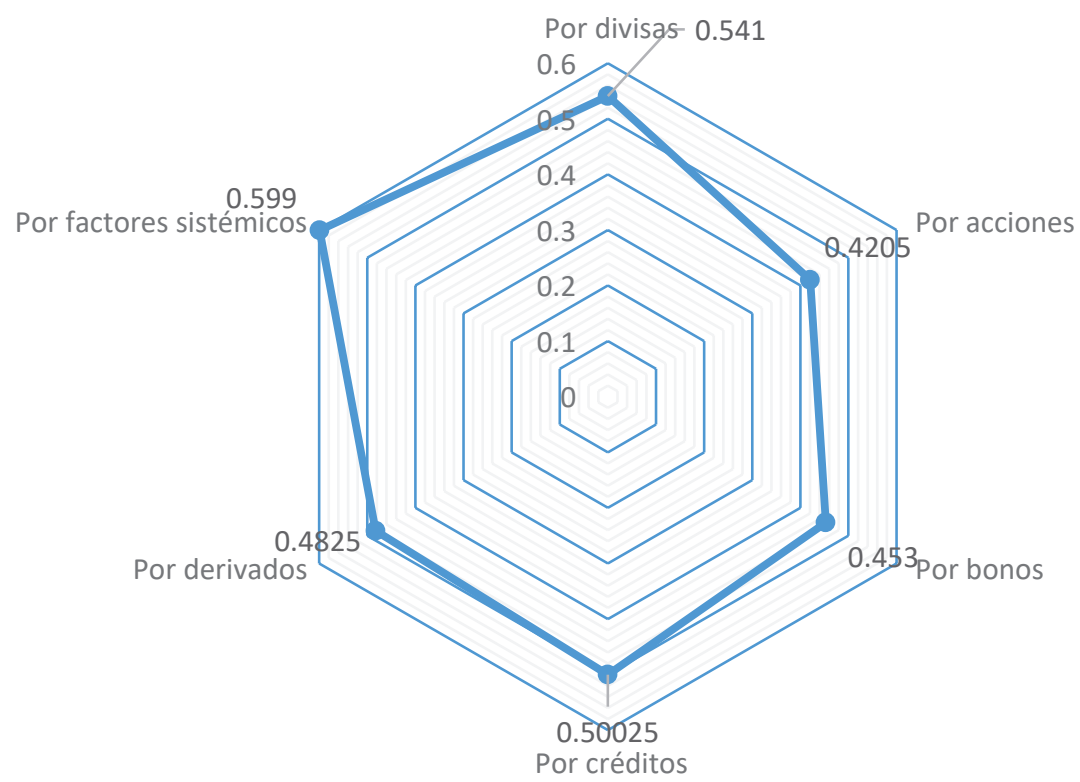

Figura 2. Incidencia del riesgo de Mercado

Fuente: Aplicación de encuesta. Elaboración Propia

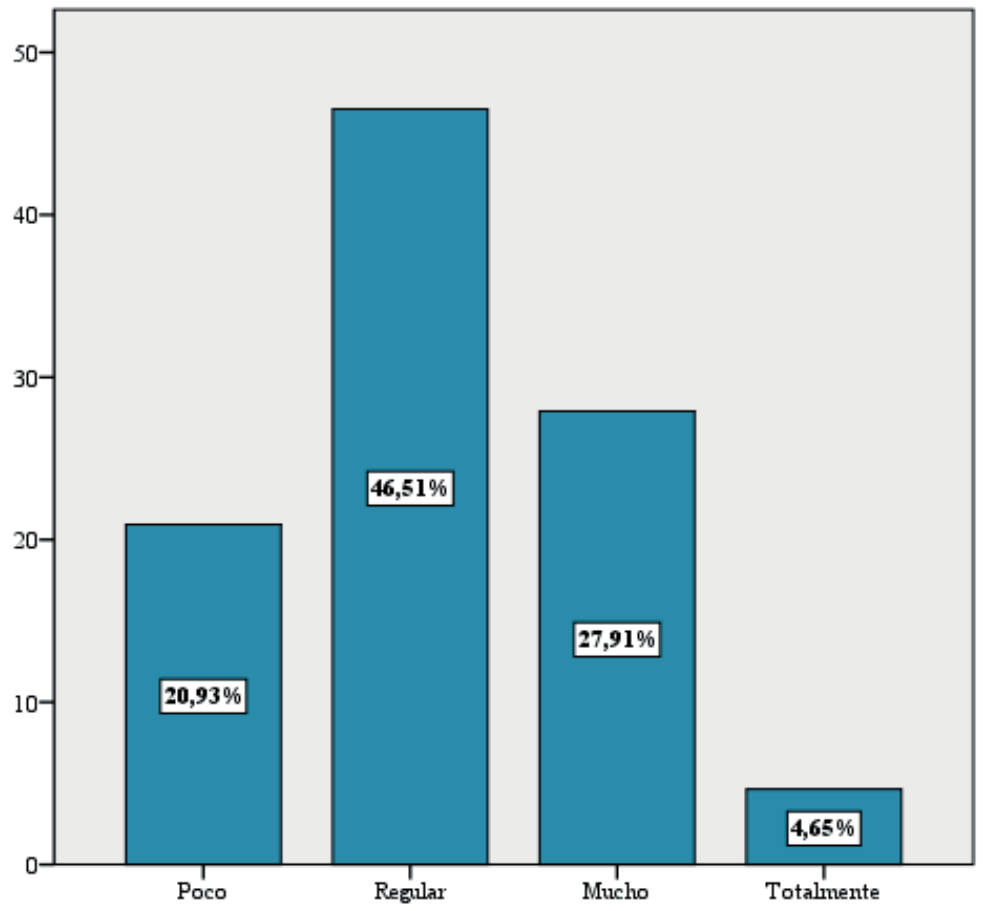

Figura 3. Riesgo de Mercado relacionado a divisas.

Fuente: Aplicación de encuesta. Elaboración Propia 
componente del SGRM de mayor incidencia en la estabilidad de los bancos.

La figura 4 nos muestra que el 44,19\% de los encuestados considera que este riesgo de acciones es Regular, segunda de un 32,56\% de quienes consideran que este es Poco, en tanto que el 18,60\% muestran que esta es Mucho y solo el 4,65\% opinan que no existe relación (Nada). Es el componente del SGRM de menor incidencia en la estabilidad bancaria, lo cual se puede relacionar con la volatilidad propia del mercado bursátil.

Los resultados mostrados en la figura 5, nos permite afirmar que el 37,21\% de los encuestados consideran que esta es Regular, seguida de un $34,88 \%$ de quienes consideran que este riesgo relacionado a los bonos es Poco, en un 20,93\% consideran que este riesgo es Mucho, un 4,65\% consideran que este riesgo es nulo o Nada y solo el 2,33\% manifestaron que este riesgo relacionado a bonos es Total.

La figura 6 muestra que el 32,56 \% de la muestra considera que es Mucho o Regular el riesgo en cuanto a los créditos, el 27,91\% manifestaron que este riesgo es Poco, el 4,65\% manifestaron que dicho riesgo es nulo o Nada, y solo el $42,33 \%$ manifestaron que el riesgo de mercado relacionado a créditos es Total.
En la evaluación correlativa de las variables (gestión de los riesgos bancarios de mercado y estabilidad de las empresas bancarias), se puede observar que existe un índice de correlación del modelo de $\mathrm{r}$ de Pearson con un valor de 0,97 ptos., en tanto que el índice de significancia es de 0,021 ptos., el mismo que es inferior al 0,05 exigido para la validez del supuesto de que la Gestión de Riesgo de Mercado conforme a las normas de gestión de riesgos de la SBS influye en la estabilidad de las empresas bancarias.

La figura 7 nos indica que el impacto de la Gestión del Riesgo de Mercado respecto a los factores sistémicos se percibe menor. El 27,3\% de los encuestados consideran el impacto de dicho riesgo relacionado a los factores sistémicos es Total; 27,3\% consideran que este riesgo es Poco, el 25,58\% consideran que este riesgo es Regular, el 16,28\% o Mucho y solo el 4,55\% manifestaron que este riesgo es nulo o nada.

\section{DISCUSIÓN}

Dado que la importancia de la gestión de riesgos de mercado se ha acrecentado en los años recientes, debido a la creciente volatilidad de los mercados financieros, y su inclusión en la normativa es aún incipiente, no están disponibles

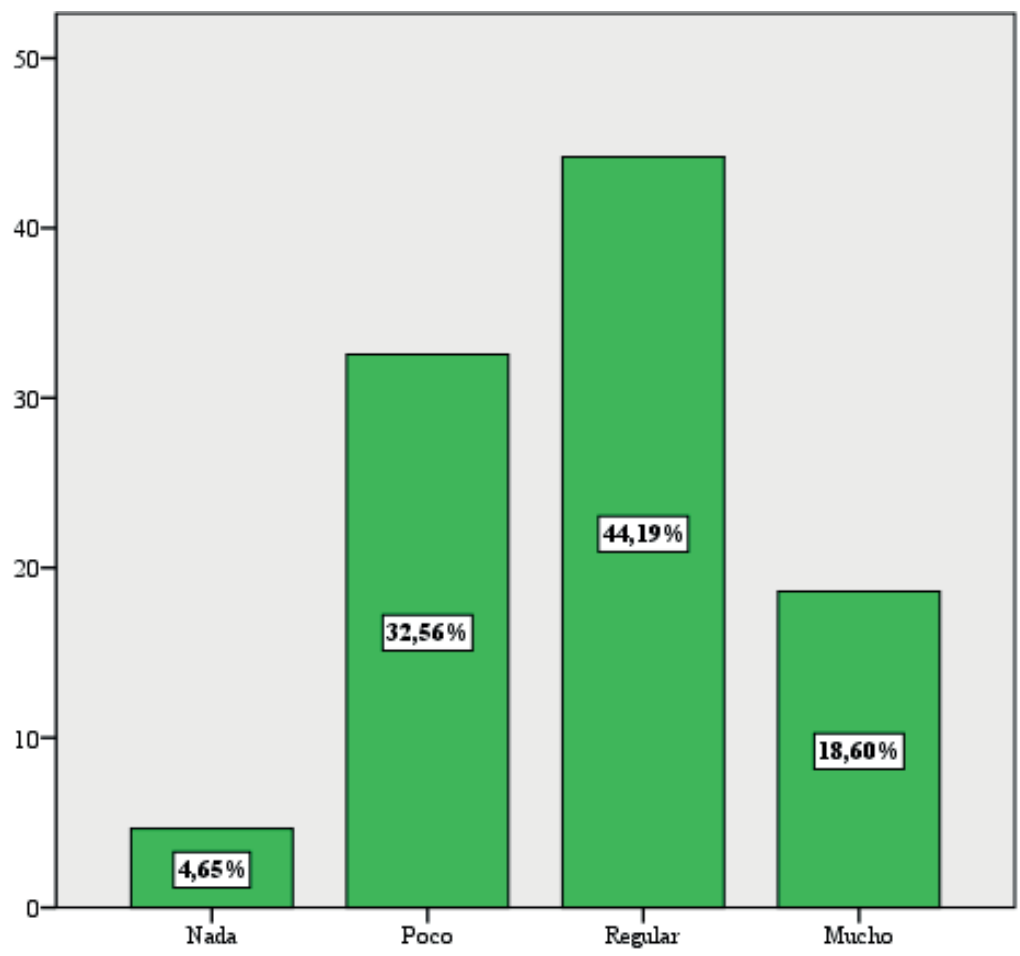

Figura 4. Riesgo de Mercado relacionado a acciones

Fuente: Aplicación de encuesta. Elaboración Propia 


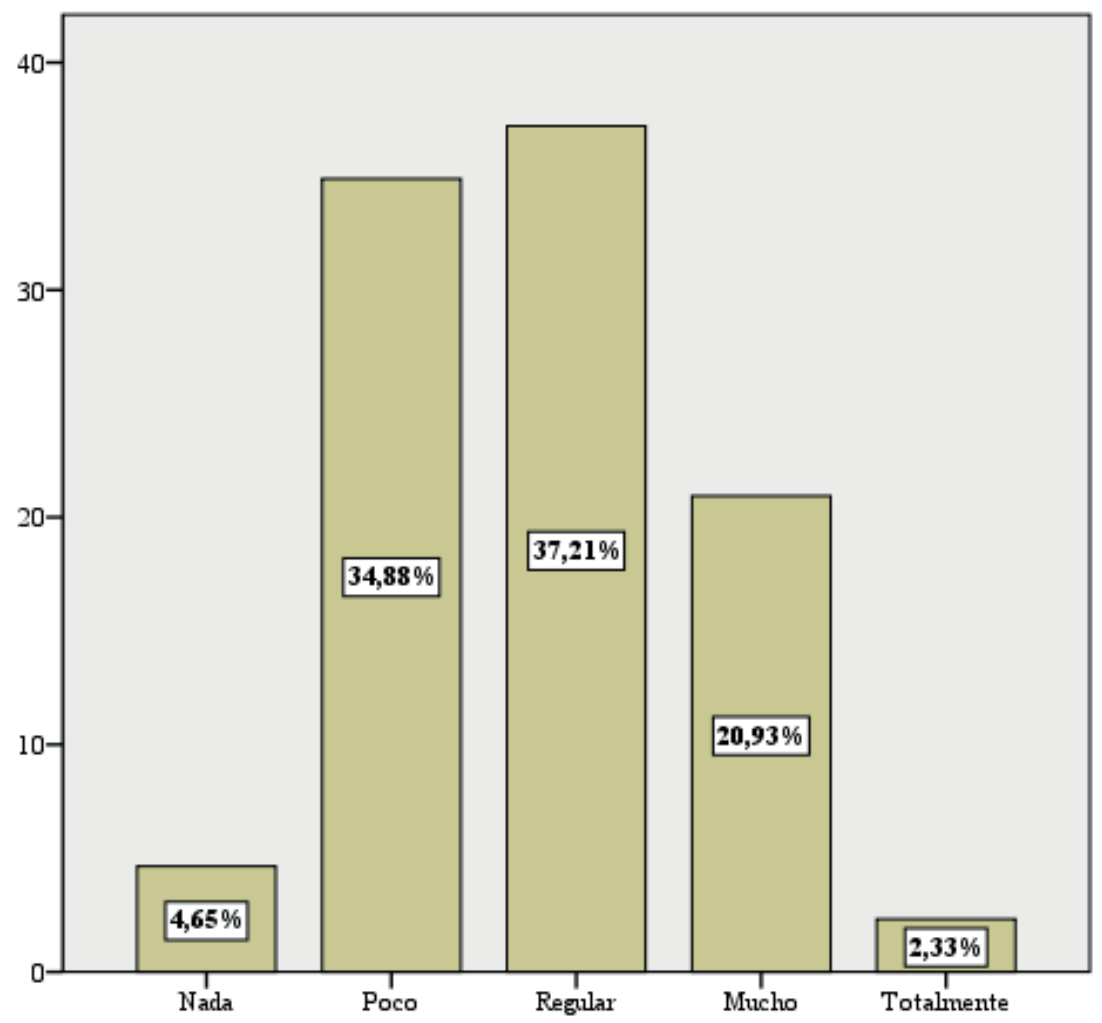

Figura 5. Riesgo de Mercado relacionado a bonos

Fuente: Aplicación de encuesta. Elaboración Propia

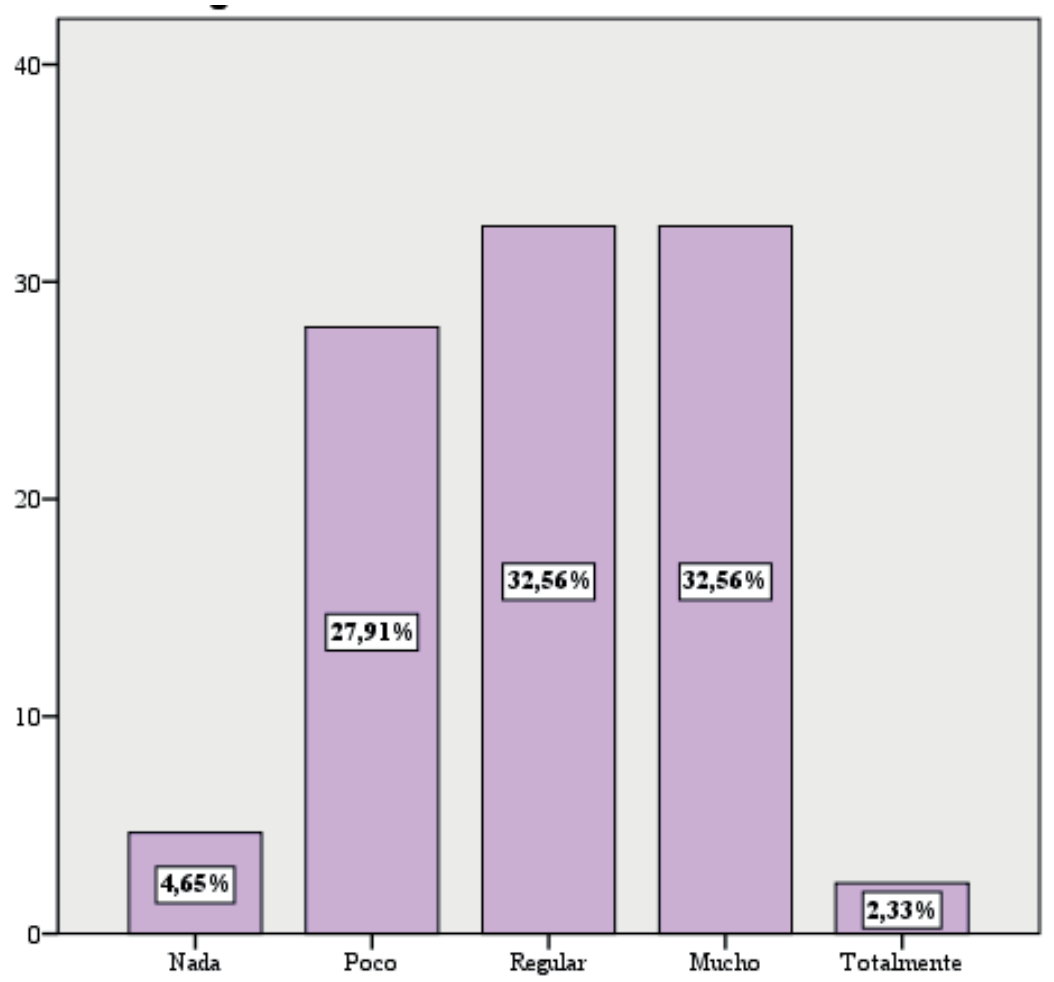

Figura 6. Riesgo de Mercado relacionado a créditos

Fuente: Aplicación de encuesta. Elaboración Propia 


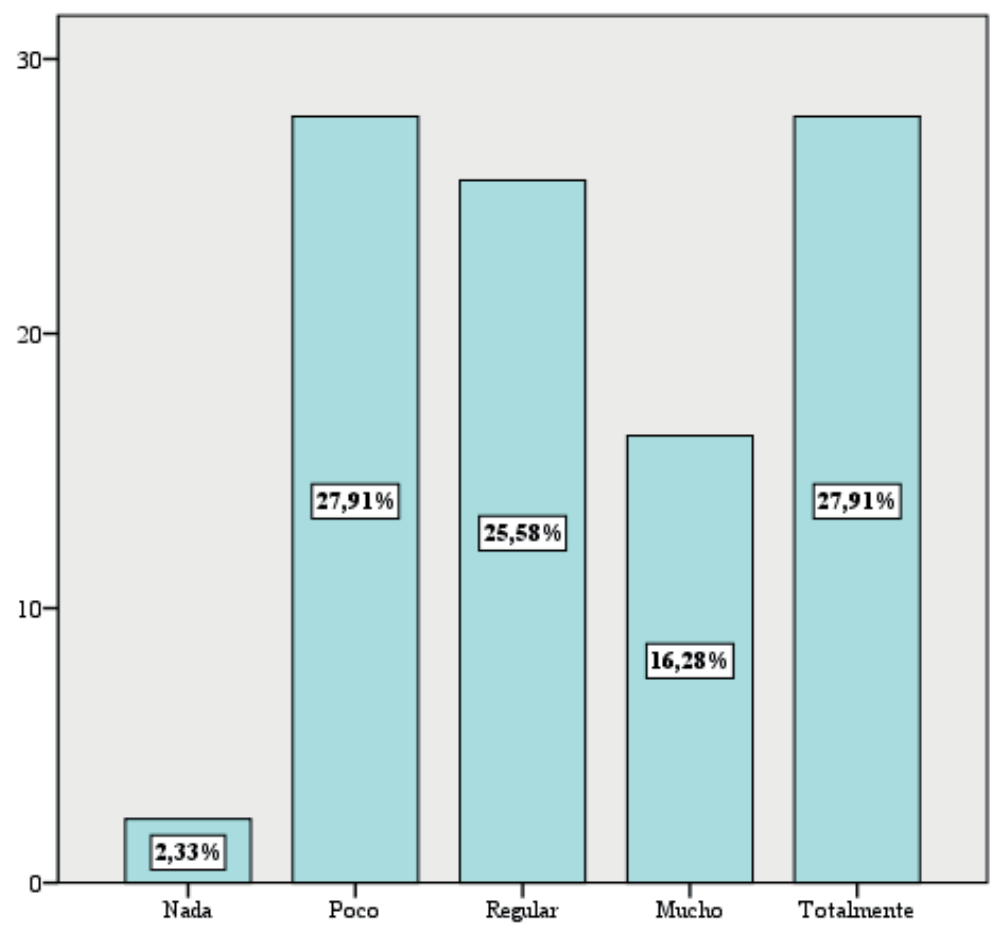

Figura 7. Riesgo de mercado relacionado a factores sistémicos

Fuente: Aplicación de encuesta. Elaboración Propia

estudios sobre el SGRM y la estabilidad del sistema bancario, lo cual hace necesario comparar los resultados de la investigación con el marco teórico y normativo especificado, esperando motivar a otros investigadores a tratar el tema desde diferentes contextos nacionales y con enfoques metodológicos. Si la aplicación del SGRM, tal como se produce en el Perú, fuera la más adecuada para la estabilidad del sistema bancario, el índice de correlación se acercaría a 1, lo cual claramente no sucede. Ello indica que las disposiciones planteadas son relevantes, pero existen otros factores externos potenciales no contemplados o contemplados insuficientemente en las normas, que están afectando la eficiencia del sistema de gestión de riesgos de mercado, normas aplicadas por la SBS conforme a los Acuerdos de Capital de Basilea. En este sentido, es útil la comparación de estos resultados con lo concluido por Saldaña (2009), en relación a las características del sistema bancario peruano, quien ha recalcado la característica de concentración, calificándola de oligopólica. Asimismo, Durand (2017) aporta el concepto de grupos de poder económico, coincidiendo que 3 de los 12 grupos mencionados están ligados a 3 de los 4 mayores bancos peruanos, en especial al Grupo Crédito (Banco de Crédito del Perú), Grupo Intercorp (Interbank) y Grupo Brescia (BBVA). La pertinencia del SGRM y su impacto en la estabilidad del sistema bancario es regular e influye en ella positivamente, pero puede ser afectada por aspectos como la concentración de créditos, activos y patrimonio en el sistema bancario peruano, la presencia de grupos de poder económico en los bancos más grandes del sistema, el posible abuso de esta concentración con esquemas de operaciones vinculadas, la segmentación del mercado y de la morosidad en el sistema, la limitación de la falta de una supervisión macroprudencial, y la gran incidencia de las operaciones de derivados financieros y esquemas de ingeniería financiera, difíciles de supervisar y que incrementan el riesgo.

\section{REFERENCIAS}

Alva, J. y Rivas, V. (2012). El reordenamiento del sistema bancario peruano. Lima: Fondo Editorial de la Universidad de San Martin de Porres.

Alva, M. (2016). Informe del BCR, El 27\% de las familias peruanas está en situación de sobreendeudamiento. Diario Gestión, p. 2.

Apostolik, R., Donohue, C. y Went, P. (2011). Fundamentos del riesgo bancario y regulación. Madrid: GARP y Delta Publicaciones.

Banco Central de Reserva del Perú. (2017). Reporte de Estabilidad Financiera BCRP - Mayo 2017. Lima: BCRP. 
Brealey, R., Myers, S. y Allen, F. (2015). Principios de Finanzas corporativas. México: McGraw Hill.

Castro, E. (2011). Riesgos a los que se enfrentan las entidades bancarias en el Perú (Tesis para optar el grado de Magister en Derecho. Lima: Universidad Nacional Mayor de San Marcos.

Comité de Supervisión Bancaria de Basilea. (2001). Nuevo Acuerdo de Capital de Basilea. Basilea: BIS.

Comité de Supervisión Bancaria de Basilea. (2018). Principios básicos de la Supervisión bancaria eficaz. Recuperado de Banco de Pagos Internacionales: https:// www.bis.org/publ/bcbs230_es.pdf

Congreso de los Estados Unidos (2011). Informe de Investigación de la crisis financiera, Informe Final de la Comisión Nacional Investigadora sobre las causas de la crisis financiera en los Estados Unidos, del Congreso de los Estados Unidos. Recuperado de Finantial Crisis Inquiry Commission: http://www.fcic.gov/report/ conclusions

Durand, F. (2017). Los doce apóstoles de la economía peruana. Una mirada social a los grupos de poder, limeños y provincianos. Lima: Fondo Editorial Pontificia Universidad Católica del Perú.

Flores, A. e Hidalgo., M. (2011). Desregulación Financiera y Crisis Económica Global. Quipukamayoc. Revista de la Facultad de Ciencias Contables. 19(36), 147-155.

Fondo Monetario Internacional. (2013). Informe de Estabilidad Financiera Mundial. Washington D.C.: Fondo Monetario Internacional.

Freixas, X. (1990). Fundamentación teórica de la regulación de los mercados financieros. Revista Moneda y Crédito. 190

Gutiérrez, C. (2013). Evolución e impacto de la regulación bancaria internacional hasta Basilea III: El caso de América Latina. Pecvnia, 147 - 173.

Holand, G. (2016). Basilea: nuevos paradigmas. Herramientas para gerencias los riesgos bancarios y financieros internacionales. Buenos Aires: EDICON.

Huertas, D. (2015). La Colocación De Créditos MYPES y la relación con el nivel de morosidad en el sistema bancario peruano del 2010 al 2014. Lima, Lima, Perú: Tesis Profesional. Universidad de San Martin de Porres.

Ley 26702, L. N. (1996). Ley General del Sistema Financiero y del Sistema de Seguros y Ley Orgánica de la Superintendencia de Banca y Seguros. Lima, Perú: El Peruano.
Lizarzaburu, E., Berggrun, L., y Quispe, J. (2012). Gestión de riesgos financieros. Experiencia de un banco latinoamericano. Estudios Gerenciales, 28(125), 96 -103. doi:https://doi.org/10.1016/S0123-5923(12)70012-8

López, P. (2018). Implicaciones Económicas y Financieras de la Regulación de Basilea en el Sector Bancario Mexicano: Un análisis de riesgo de crédito y de mercado (Tesis doctoral en economía). Ciudad de México: Universidad Nacional Autónoma de México.

Marín, J. y Valle, C. (2009). La Regulación y Supervisión del Sistema Financiero Ante la Crisis Económica. Boletín de Estudios Económicos. 64(198), 441-468.

Mishkin, F. (2014). Moneda, banca y mercados financieros. 10. ${ }^{\mathrm{a}}$ ed. México D.F.: Editorial Pearson.

Manrique, O. (2018). Banca tiene colchón de capital extra de US\$ 5,100 millones ante eventual crisis. Gestión. Lima, Perú.

Morales, J. (2014). Comportamiento del sistema bancario y sus efectos en la economía y las finanzas (Tesis doctoral) Lima, Perú: Universidad de San Martin de Porres.

Partal, A., y Gómez, P. (2011). Gestión de riesgos financieros en la banca internacional. Madrid: Pirámide.

Reinhart, C. y Rogoff, K. (2011). Esta vez es distinto: ocho siglos de necedad financiera. México D.F.: Fondo de Cultura Económica.

Saldaña, J. (2009). El comportamiento de la economía y la gestión de riesgos de los intermediarios financieros bancarios: caso Perú: 2001 - 2006 (Tesis para optar el grado Académico de Magister en Contabilidad con mención en Banca y Finanzas).

Superintendencia de Banca, Seguros y AFP. (2017). Resolución SBS No 272- 2017. Reglamento de Gobierno Corporativo y de la Gestión Integral de Riesgos. Diario Oficial El Peruano. Lima, Perú

Superintendencia de Banca, Seguros y AFP. (2017). Boletín Estadístico. Lima: SBS.

Superintendencia de Banca, Seguros y AFP. (2017). Reglamento para la gestión de riesgo de mercado. Lima: SBS.

Wolf, M. (2015). La gran crisis. Cambios y consecuencias. Barcelona: Deusto. 